ARTICLE

\title{
A national cohort study (2000-2018) of long-term air pollution exposure and incident dementia in older adults in the United States
}

\author{
Liuhua Shi (10 1,9凶 , Kyle Steenland ${ }^{1,9}$, Haomin $\mathrm{Li}^{2}$, Pengfei Liu ${ }^{3}$, Yuhan Zhang ${ }^{2}$, Robert H. Lyles ${ }^{4}$,
} Weeberb J. Requia (1) ${ }^{5}$, Sindana D. Ilango ${ }^{6}$, Howard H. Chang ${ }^{1,4}$, Thomas Wingo (1) ${ }^{7}$, Rodney J. Weber ${ }^{3}$ \& Joel Schwartz (iD ${ }^{8}$

Air pollution may increase risk of Alzheimer's disease and related dementias (ADRD) in the U.S., but the extent of this relationship is unclear. Here, we constructed two national U.S. population-based cohorts of those aged $\geq 65$ from the Medicare Chronic Conditions Warehouse (2000-2018), combined with high-resolution air pollution datasets, to investigate the association of long-term exposure to ambient fine particulate matter $\left(\mathrm{PM}_{2.5}\right)$, nitrogen dioxide $\left(\mathrm{NO}_{2}\right)$, and ozone $\left(\mathrm{O}_{3}\right)$ with dementia and $A D$ incidence, respectively. We identified $\sim 2.0$ million incident dementia cases $(N=12,233,371$; dementia cohort $)$ and $\sim 0.8$ million incident $A D$ cases $(N=12,456,447 ; A D$ cohort). Per interquartile range (IQR) increase in the 5-year average $\mathrm{PM}_{2.5}\left(3.2 \mu \mathrm{g} / \mathrm{m}^{3}\right), \mathrm{NO}_{2}(11.6 \mathrm{ppb})$, and warm-season $\mathrm{O}_{3}(5.3 \mathrm{ppb})$ over the past 5 years prior to diagnosis, the hazard ratios (HRs) were 1.060 (95\% confidence interval [Cl]: 1.054, 1.066), 1.019 (95\% Cl: 1.012, 1.026), and 0.990 (95\% Cl: $0.987,0.993)$ for incident dementias, and 1.078 (95\% Cl: 1.070, 1.086), 1.031 (95\% Cl: 1.023, 1.039), and 0.982 (95\% Cl: $0.977,0.986)$ for incident $A D$, respectively, for the three pollutants. For both outcomes, concentration-response relationships for $\mathrm{PM}_{2.5}$ and $\mathrm{NO}_{2}$ were approximately linear. Our study suggests that exposures to $\mathrm{PM}_{2.5}$ and $\mathrm{NO}_{2}$ are associated with incidence of dementia and AD.

\footnotetext{
${ }^{1}$ Gangarosa Department of Environmental Health, Rollins School of Public Health, Emory University, Atlanta, GA, USA. ${ }^{2}$ Department of Epidemiology, Rollins School of Public Health, Emory University, Atlanta, GA, USA. ${ }^{3}$ School of Earth and Atmospheric Sciences, Georgia Institute of Technology, Atlanta, GA, USA. ${ }^{4}$ Department of Biostatistics and Bioinformatics, Rollins School of Public Health, Emory University, Atlanta, GA, USA. ${ }^{5}$ School of Public Policy and Government, Fundação Getúlio Vargas, Brasília, DF, Brazil. ${ }^{6}$ Department of Epidemiology, School of Public Health, University of Washington, Seattle, WA, USA. ${ }^{7}$ Department of Neurology and Human Genetics, School of Medicine, Emory University, Atlanta, GA, USA. ${ }^{8}$ Department of Environmental Health, Harvard T.H. Chan School of Public Health, Boston, MA, USA. ${ }^{9}$ These authors contributed equally: Liuhua Shi, Kyle Steenland. ${ }^{\circledR}$ email: liuhua.shi@emory.edu
} 
D ementia is a major public health issue, affecting $>47$ million people worldwide ${ }^{1}$. Alzheimer's disease (AD) contributes to about two-thirds of dementia cases and is the sixth leading cause of death in the United States ${ }^{2}$. In response, the National Alzheimer's Project Act was signed into law to overcome dementia, and the National Plan was launched with Goal 1 aiming to prevent and effectively treat dementia (delay onset, slow progression) by $2025^{3}$. As there are no diseasemodifying treatments for the most common types of dementia, it is a top research priority to identify modifiable risk factors for dementia that can be intervened on at the population level.

There is growing evidence associating air pollution with neurodegenerative disease. A systematic review by Peters et al. ${ }^{4}$ found nine longitudinal studies of air pollution and $\mathrm{AD}$ and related dementias (ADRD). Among them, five of six showed a positive association between increased exposure to $\mathrm{PM}_{2.5}$ and dementia or $\mathrm{AD}$; four of four showed an association between $\mathrm{NO}_{2}$ and dementia or $\mathrm{AD}$, whereas one of three did so for ozone $\left(\mathrm{O}_{3}\right)$. Fu and Yung ${ }^{5}$ published a review and meta-analysis of $\mathrm{AD}$ and air pollution, and found a twofold excess risk of $A D$ for a $10 \mu \mathrm{g} / \mathrm{m}^{3}$ increase of $\mathrm{PM}_{2.5}$ among six studies, and no increased risk for $\mathrm{NO}_{2}$ in four studies, nor for $\mathrm{O}_{3}$ in three studies. There have been several longitudinal studies since these reviews, with the majority finding positive associations between air pollutants and either dementia or $\mathrm{AD}^{6-14}$. A few of these studies examine the associations in US populations, and these studies have almost exclusively used hospitalization as a measure of morbidity $6,7,11,13$. The diagnosis of ADRD, however, likely occurs in doctor visits, and ADRD does not generally result in hospitalizations. Thus, hospitalization records may not well represent either disease incidence or prevalence in the overall population, and likely leads to an underestimation of the number of cases, and unclear generalizability. In addition, neuropathologic changes are known to occur many years prior to the diagnosis ${ }^{15}$, and the relevant time window in which air pollution might increase the risk of dementia or $\mathrm{AD}$ is unclear.

To address these knowledge gaps in studying ADRD incidence in the US, here we constructed a national, population-based cohort study from Medicare data to investigate the impact of long-term exposure to $\mathrm{PM}_{2.5}, \mathrm{NO}_{2}$, and warm-season (May to October) $\mathrm{O}_{3}$ on dementia and $\mathrm{AD}$ incidence. To better measure disease incidence, we required a 5-year "clean" period without events of interest after enrollment in Medicare system and used all Medicare claims nationwide (2000-2018), including inpatient and outpatient claims, carrier file (primarily doctor visits), skilled nursing facility, and home health-care claims to identify the first diagnosis of ADRD. We used high-resolution $(1 \mathrm{~km} \times 1 \mathrm{~km})$ daily surface-level concentration fields of $\mathrm{PM}_{2.5}, \mathrm{NO}_{2}$, and $\mathrm{O}_{3}$ for 2000-2016, estimated based on ground observations, satellite data, chemical transport modeling, land use, and meteorological data using national spatiotemporal ensemble exposure models ${ }^{16-18}$. We assigned air pollution exposure to subjects based on resident ZIP code, and calculated time-varying 5-year lagged moving averages for each follow-up year.

\section{Results}

Study population characteristics. Table 1 provides descriptive information on the dementia cohort and $\mathrm{AD}$ cohort. Both cohorts were followed after requiring a 5-year period without events of interest to better capture disease incidence. There were 12.2 and 12.4 million people in dementia and $\mathrm{AD}$ cohorts, respectively (Table 1). Most of the studied subjects $(78.5 \%$ and $78.1 \%$ for dementia and $\mathrm{AD}$, respectively) entered the cohorts between ages 65 and 74. The median follow-up was 7 years in both cohorts. More than $90 \%$ were not eligible for Medicaid, indicating that
Table 1 Descriptive statistics for the study population.

\begin{tabular}{|c|c|c|c|c|}
\hline \multirow[b]{2}{*}{ Variables } & \multicolumn{2}{|c|}{ Dementia cohort } & \multicolumn{2}{|l|}{ AD cohort } \\
\hline & Number & $\%$ & Number & $\%$ \\
\hline Number of events & $2,025,130$ & 16.6 & 804,668 & 6.5 \\
\hline $\begin{array}{l}\text { Number of the total } \\
\text { population }\end{array}$ & $12,233,371$ & 100 & $12,456,447$ & 100 \\
\hline Total person-years & $89,035,081$ & 100 & $93,278,266$ & 100 \\
\hline \multirow{2}{*}{\multicolumn{5}{|c|}{ Age at entry (years) }} \\
\hline & & & & \\
\hline $65-74$ & $9,597,788$ & 78.5 & $9,734,481$ & 78.1 \\
\hline $75-114$ & $2,635,583$ & 21.5 & $2,721,966$ & 21.9 \\
\hline \multicolumn{5}{|l|}{ Sex } \\
\hline Male & $5,023,879$ & 41.1 & $5,107,942$ & 41.0 \\
\hline Female & $7,209,492$ & 58.9 & $7,348,505$ & 59.0 \\
\hline \multicolumn{5}{|l|}{ Race } \\
\hline White & $11,023,202$ & 90.1 & $11,214,287$ & 90.0 \\
\hline Black & 649,081 & 5.3 & 666,619 & 5.4 \\
\hline Other ${ }^{a}$ & 561,088 & 4.6 & 575,541 & 4.6 \\
\hline \multicolumn{5}{|l|}{ Medicaid eligibility } \\
\hline Dual-eligible & 800,139 & 6.5 & 852,499 & 6.8 \\
\hline Non-dual eligible & $11,433,232$ & 93.5 & $11,603,948$ & 93.2 \\
\hline \multicolumn{5}{|l|}{ Comorbidity } \\
\hline Diabetes & $4,433,314$ & 36.2 & $4,590,000$ & 36.8 \\
\hline Hypertension & $10,273,506$ & 84.0 & $10,502,180$ & 84.3 \\
\hline Stroke & $1,991,730$ & 16.3 & $2,137,239$ & 17.2 \\
\hline Heart failure & $3,388,540$ & 27.7 & $3,598,028$ & 28.9 \\
\hline No comorbidities $^{b}$ & $1,642,674$ & 13.4 & $1,865,751$ & 15.0 \\
\hline \multicolumn{5}{|l|}{ Air pollutants ${ }^{\mathrm{C}}$} \\
\hline Annual $\mathrm{PM}_{2.5}\left(\mu \mathrm{g} / \mathrm{m}^{3}\right)$ & $9.3(3.2)$ & $9.3(3.2)$ & & \\
\hline Annual $\mathrm{NO}_{2}(\mathrm{ppb})$ & $17.1(11.6)$ & $17.1(11.6)$ & & \\
\hline Warm-season $\mathrm{O}_{3}(\mathrm{ppb})$ & $42.6(5.3)$ & $42.6(5.3)$ & & \\
\hline
\end{tabular}

most were defined as being above the poverty level ${ }^{19}$. A majority of the study population had comorbidity at some point during follow-up. 16.6\% developed dementia ( 2.0 million cases), $6.5 \%$ developed AD ( $\sim .8$ million cases), and Supplementary Table 1 presents detailed demographic information for the cases and noncases.

Air pollution levels. The average annual level of $\mathrm{PM}_{2.5}$ of cohort participants during the study period, $9.3 \mu \mathrm{g} / \mathrm{m}^{3}$, was below the US EPA standard of $12 \mu \mathrm{g} / \mathrm{m}^{3}$; The average $\mathrm{NO}_{2}$ level was $17.1 \mathrm{ppb}$, considerably below the EPA annual standard of $\mathrm{NO}_{2}$ of $53 \mathrm{ppb}$. The annual warm-season average $\mathrm{O}_{3}$ was $42.6 \mathrm{ppb}$. EPA does not have a standard for annual warm-season $\mathrm{O}_{3}$. As a reference, the EPA standard for daily maximum of 8-hour average $\mathrm{O}_{3}$ is $70 \mathrm{ppb}$ (Table 1). We examined warm-season $\mathrm{O}_{3}$, because $\mathrm{O}_{3}$ is more readily formed in the warm season ${ }^{20}$, and this metric is often used in long-term epidemiological studies ${ }^{21}$. Fig. 1 shows the distribution of the three pollutants across the US during our study period, as estimated by the exposure models used in our analysis. $\mathrm{PM}_{2.5}$ is highest in the eastern US and in California, $\mathrm{O}_{3}$ in the West, and $\mathrm{NO}_{2}$ (largely produced by traffic) in urban centers. Further detail on exposure levels can be found in Supplementary Table 2 . The three pollutants in our data were only modestly correlated. The Pearson correlations between pollutants (average exposure within the past 5 years) were as follows: $\mathrm{PM}_{2.5}$ and $\mathrm{O}_{3}$ $0.22, \mathrm{NO}_{2}$ and $\mathrm{O}_{3} 0.19$, and $\mathrm{NO}_{2}$ and $\mathrm{PM}_{2.5}$ 0.39.

Health effect estimates. Fig. 2 provides the main study results from the Cox proportional hazards models stratified by individual characteristics, adjusting for neighborhood-level socioeconomic 
a

b
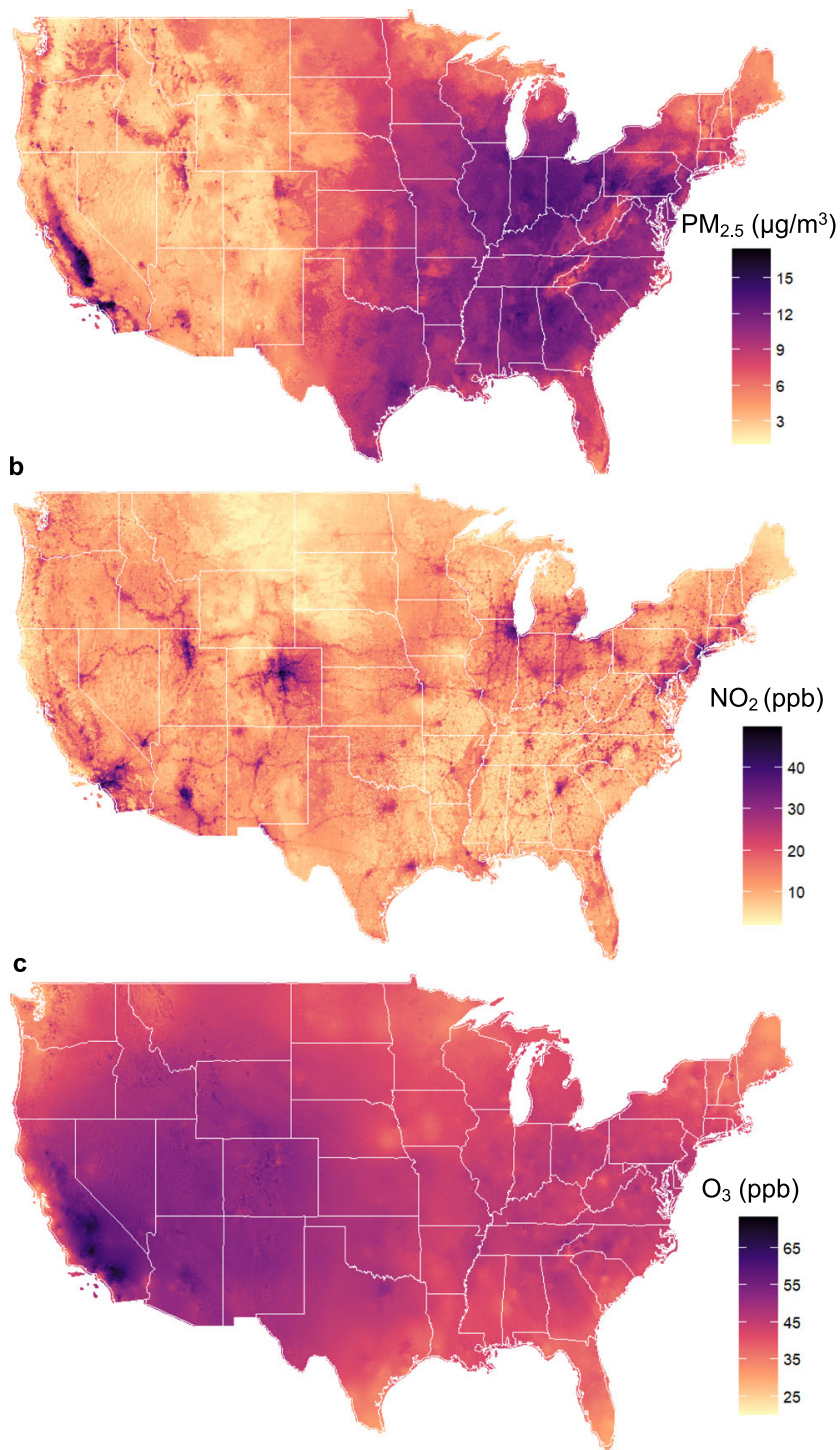

Fig. 1 Maps of the spatial distributions of air pollutants studied. The three panels present the average concentrations of $\mathbf{a}$ annual $\mathrm{PM}_{2.5}\left(\mu \mathrm{g} / \mathrm{m}^{3}\right)^{16}$, b annual $\mathrm{NO}_{2}(\mathrm{ppb})^{17}$, and $\mathbf{c}$ warm-season $\mathrm{O}_{3}(\mathrm{ppb})^{18}$ at $1-\mathrm{km}^{2}$ resolution across the contiguous United States over the study period, respectively. Map was made from the census bureau shapefile (cb_2017_us_county_500k.shp, https://www2.census.gov/geo/tiger/GENZ2017/shp/) using R software, and no licenses are required as this map was provided free of any copyright restrictions. Source data are provided as a Source Data file.

status (SES) (see details in Methods), behavioral risk factors, health-care capacity variables, and residual temporal and spatial trends (see Methods). An interquartile range (IQR) increase in the 5-year average of the annual $\mathrm{PM}_{2.5}\left(3.2 \mu \mathrm{g} / \mathrm{m}^{3}\right)$ in the 5 years prior to diagnosis was associated with an increased risk of dementia $(\mathrm{HR}=1.061,95 \% \mathrm{CI}: 1.056,1.067)$ in single-pollutant models, which changes little in models with other pollutants. An IQR increase in 5-year average $\mathrm{NO}_{2}(11.6 \mathrm{ppb})$ is associated with an HR of 1.035 (95\% CI: 1.028, 1.042) in single-pollutant models, dropping to 1.019 (95\% CI: $1.012,1.026)$ in multi-pollutant models. An IQR increase in the 5-year average of warm-season $\mathrm{O}_{3}(5.3 \mathrm{ppb})$ has little effect on dementia rates, with HRs of 1.002 $(95 \%$ CI: $0.998,1.005)$ in single-pollutant models and $0.990(95 \%$ CI: $0.987,0.993)$ in multi-pollutant models.
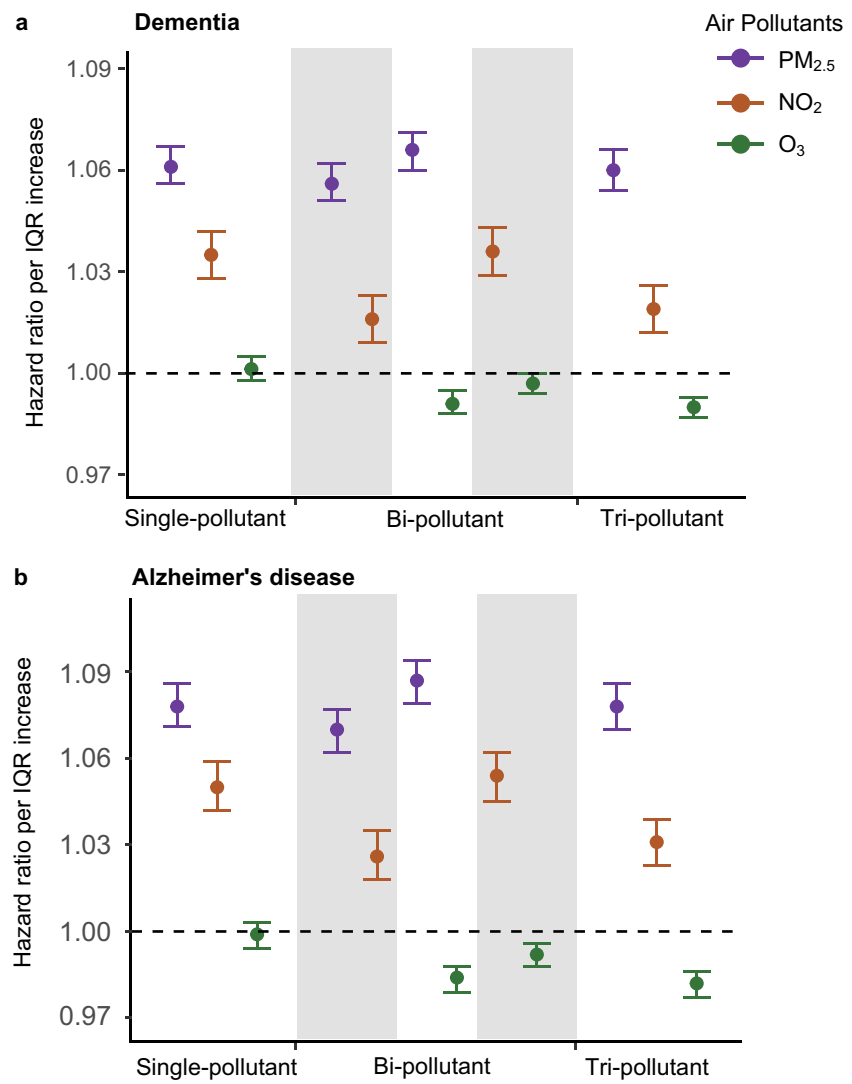

Fig. 2 Results of the Cox proportional hazards models. The two panels present the hazard ratios of a dementia ( $n=12,233,371$ individuals examined) or b Alzheimer's disease ( $A D, n=12,456,447$ individuals examined) associated with per IQR increase in annual $\mathrm{PM}_{2.5}$, or annual $\mathrm{NO}_{2}$, or warm-season $\mathrm{O}_{3}$ concentration, respectively. The estimated hazard ratios were obtained using single pollutant, bi-pollutant, and tri-pollutant models. Error bars stand for the 95\% confidence intervals. The gray and white stripes are used to distinguish any two adjacent models. Source data are provided as a Source Data file.

The findings for $\mathrm{AD}$ have a similar pattern to those for dementia, but the hazard ratios (HRs) are higher per IQR increase, being 1.078 (95\% CI: 1.071, 1.086) for $\mathrm{PM}_{2.5}, 1.050$ (95\% CI: $1.042,1.059)$ for $\mathrm{NO}_{2}$, and 0.999 (95\% CI: $\left.0.995,1.003\right)$ for $\mathrm{O}_{3}$ assessing each pollutant individually. After adjusting for copollutants, the effect estimates were similar for $\mathrm{PM}_{2.5}(\mathrm{HR}=$ $1.078,95 \% \mathrm{CI}: 1.070,1.086)$ and attenuated for $\mathrm{NO}_{2}(\mathrm{HR}=$ 1.031, 95\% CI: $1.023,1.039)$, while $\mathrm{O}_{3}$ is slightly protective $(\mathrm{HR}=0.982,95 \% \mathrm{CI}: 0.977,0.987)$.

Concentration-response relationships. Figure 3 presents penalized spline curves for the three pollutants, derived from the tripollutant models. The concentration-response (C-R) relationships for $\mathrm{PM}_{2.5}$ are approximately linear for both dementia and $\mathrm{AD}$ across the exposure distribution, although for $\mathrm{AD}$ there is a suggestion of a steeper slope below $8 \mu \mathrm{g} / \mathrm{m}^{3}$. For $\mathrm{NO}_{2}$, the C-R curves for dementia and $\mathrm{AD}$ are linear for low concentrations $(<25 \mathrm{ppb})$, and then level off for higher concentrations. The curves for $\mathrm{O}_{3}$ are essentially flat for both endpoints until high, and rarely occurring concentrations. These results suggest that the adverse effects of $\mathrm{PM}_{2.5}$ and $\mathrm{NO}_{2}$ on dementia or $\mathrm{AD}$ are at least retained, if not strengthened, at low levels of air pollution exposure (e.g., below the WHO air quality guidelines: $\mathrm{PM}_{2.5} \leq$ $\left.10 \mu \mathrm{g} / \mathrm{m}^{3}, \mathrm{NO}_{2} \leq 20 \mathrm{ppb}\right)$. Across the 0.5 th to 99.5 th percentile of 


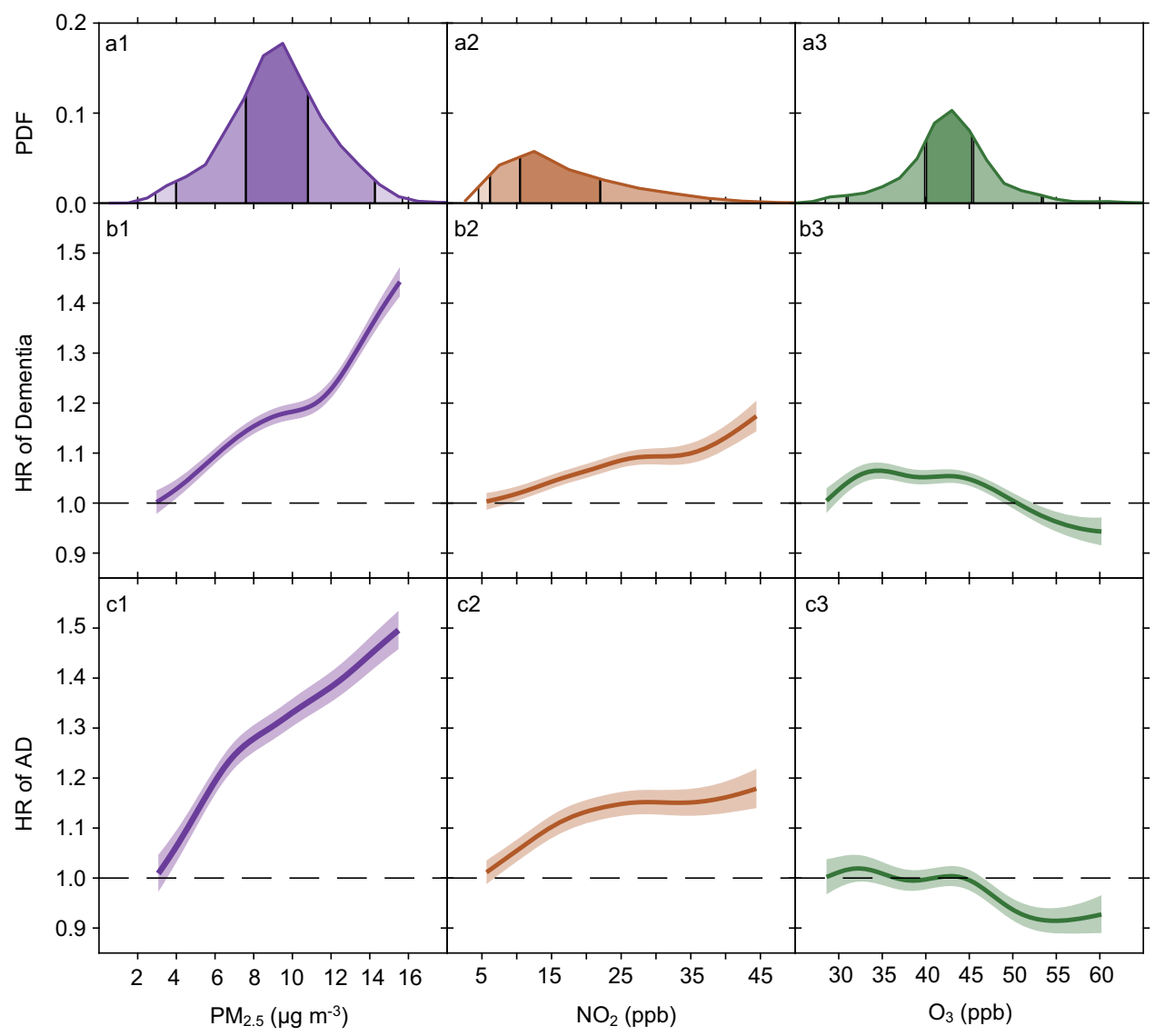

Fig. 3 Concentration-response curves. Panel. a presents the probability distribution functions (PDF) of long-term $\mathrm{PM}_{2.5}, \mathrm{NO}_{2}$, and $\mathrm{O}_{3}$ exposures; Panel b presents the concentration-response relationships between each pollutant and dementia; Panel c presents the concentration-response relationships between each pollutant and Alzheimer's disease (AD). The concentration-response curves, derived from the tri-pollutant models, are shown for the concentration ranges between 0.5 th to 99.5 th percentiles of the pollutants, i.e. with $1 \%$ poorly constrained extreme values excluded. Shading areas (from the darkest to the lightest) in a represent pollutant concentration ranges of the IQR (i.e., 25th to 75th percentiles), 95\% (2.5th to 97.5th), and 99\% (0.5th to 99.5th), respectively. Source data are provided as a Source Data file.

the exposure distribution, $\mathrm{PM}_{2.5}$ shows the strongest effect on dementia or AD among all pollutants.

Effect modifications. We examined five potential effect modifiers (sex, race (white, Black, other), Medicaid eligibility, urbanicity (expressed in quartiles of population density), and age $(<75, \geq 75)$. Fig. 4 shows HRs in each subgroup, based on the interaction term between exposure and the potential effect modifier. Most marked results were seen for an increased hazard of dementia and $\mathrm{AD}$ for Black individuals compared with white individuals in relation to both $\mathrm{PM}_{2.5}$ and $\mathrm{NO}_{2}$; a similar pattern was found for those eligible for Medicaid. At the same time, those living in the rural areas (i.e., lowest quartile of population density) were found to have notably lower effect estimates between both dementia and $\mathrm{AD}$ and both $\mathrm{PM}_{2.5}$ and $\mathrm{NO}_{2}$. Regarding age, those $<75$ had a markedly stronger association between dementia and both $\mathrm{PM}_{2.5}$ and $\mathrm{NO}_{2}$, while the association was stronger between $\mathrm{AD}$ and both $\mathrm{PM}_{2.5}$ and $\mathrm{NO}_{2}$ among those older than 75. Finally, we found little evidence of an interaction between $\mathrm{PM}_{2.5}$ or $\mathrm{NO}_{2}$, and sex in relation to dementia or $\mathrm{AD}$. For $\mathrm{O}_{3}$, all subgroup-specific estimated HRs were below one, and the association with both endpoints was stronger among those not eligible for Medicaid or those living in rural areas. The $p$ values for testing the null hypothesis that the estimated associations are the same between subgroups classified by a subpopulation indicator are shown in Supplementary Table 3.
Sensitivity analysis. Associations between long-term exposure to $\mathrm{PM}_{2.5}, \mathrm{NO}_{2}, \mathrm{O}_{3}$, and dementia or $\mathrm{AD}$ were robust to a series of sensitivity analyses. First, a more strict "clean period" by excluding anyone who had a diagnosis for dementia or $\mathrm{AD}$ in their first 10 years of follow-up yielded results similar to the main analyses (Supplementary Table 4). Second, based on this new subcohort (with 10-year clean period), the use of alternative exposure windows (annual exposure 10, 5, 1, or 0 years prior to disease diagnosis, i.e., lags $10,5,1$, or 0 ) all support a positive association with $\mathrm{PM}_{2.5}$ and $\mathrm{NO}_{2}$, but not $\mathrm{O}_{3}$, though $\mathrm{HRs}$ varied in magnitude (Supplementary Table 4). For both outcomes, associations with $\mathrm{PM}_{2.5}$ and $\mathrm{NO}_{2}$ were attenuated with increasing lag periods. Third, the observed associations with dementia or $\mathrm{AD}$ were not mediated by nor modified by comorbidities, such as diabetes, hypertension, stroke, and heart failure (Supplementary Table 5). Fourth, to account for potential bias related to moving of the resident address, we performed analyses for subjects who did not move during the follow-up period (i.e., non-mover cohort). The results are roughly consistent with the main analysis, showing significant positive associations for $\mathrm{PM}_{2.5}$ and $\mathrm{NO}_{2}$, but not for $\mathrm{O}_{3}$, for both dementia and $\mathrm{AD}$. However, compared with the main model, the effects of $\mathrm{PM}_{2.5}$ are attenuated and the effects of $\mathrm{NO}_{2}$ are enhanced (Supplementary Table 6). At last, we assessed the effect of possible outcome misclassification in two ways, one via using a linear regression model based on rates, and the other based on prior estimates of Medicare sensitivity and specificity and estimating the true number of cases within strata. 


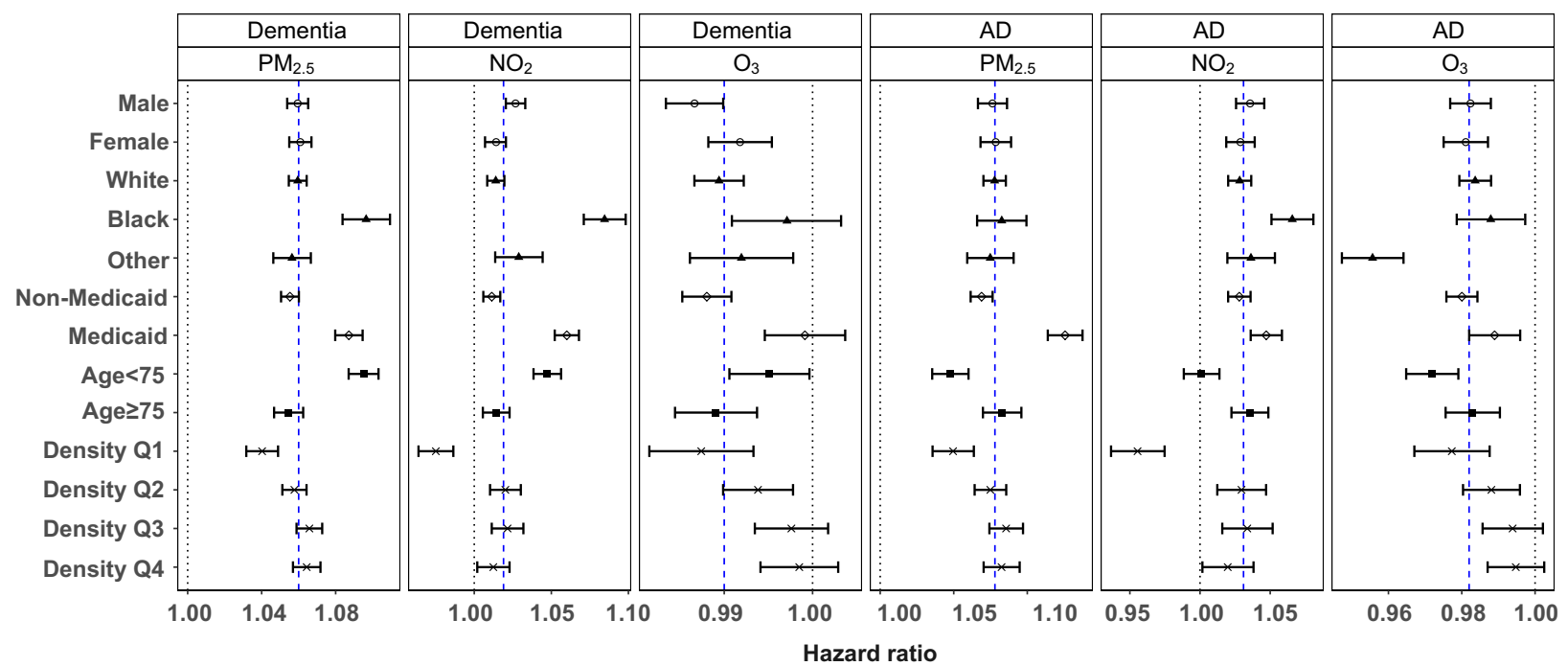

Fig. 4 Effect modifications by sex, race, Medicaid eligibility, age, and population density. Results represent the hazard ratios of dementia $(n=12,233,371$ individuals examined) or Alzheimer's disease ( $A D, n=12,456,447$ individuals examined), from the tri-pollutant models, per IQR increase in 5-year average $\mathrm{PM}_{2.5}, \mathrm{NO}_{2}$, or $\mathrm{O}_{3}$. Error bars stand for the $95 \%$ confidence intervals. The blue dashed lines indicate the overall effect estimates for all groups. "Other" includes Asian, Hispanic, American Indian or Alaskan Native, and unknown. "Density Q1-Q4" denotes quartiles of population density, i.e., low population density, low-medium population density, medium-high population density, and high population density. Source data are provided as a Source Data file.

Both methods support the findings from our main analysis, i.e., long-term exposure to $\mathrm{PM}_{2.5}$ and $\mathrm{NO}_{2}$, but not $\mathrm{O}_{3}$, were significantly associated with an increased incidence of dementia and $\mathrm{AD}$; both also suggest that misclassification has biased our findings to the null (Supplementary Tables 7 and 8).

\section{Discussion}

We found elevated HRs for both dementia and $\mathrm{AD}$ in relation to $\mathrm{PM}_{2.5}$, and less markedly to $\mathrm{NO}_{2}$, while $\mathrm{HRs}$ for warm-season $\mathrm{O}_{3}$ were not elevated. We did this study in a large US cohort (12 million), with national coverage, and including non-urban areas. For both $\mathrm{PM}_{2.5}$ and $\mathrm{NO}_{2}$, we found a larger effect on $\mathrm{AD}$ compared to dementia, which may reflect the fact that dementia includes a wide range of diseases with distinct etiologies, some of which may be unrelated to air pollution, while $\mathrm{AD}$ is a subset of dementia and a single disease, for which we found a stronger association. On the other hand, we know of no pathophysiologic data, which indicate that air pollution might affect $\mathrm{AD}$ more than dementia; data relevant to disease mechanisms would suggest both $\mathrm{AD}$ and the broader category of dementia might be affected by air pollution 22,23 .

We also found that shorter time windows between exposure $\left(\mathrm{PM}_{2.5}\right.$ or $\left.\mathrm{NO}_{2}\right)$ and disease showed slightly higher effect estimates, and we suggest (assuming the association is causal, although we acknowledge this is not possible to conclude from an observational study such as this) that this implies an acceleration of an existing process (dementia progression, i.e., accelerating cognitive decline that was already well developed). Further research assessing specific aspects of progression is warranted in the future, preferably with data on the stage of dementia at diagnosis. Moreover, our diagnosis free period requirement provides reasonable assurance that we are looking at incidence, and use of physician's visits, nursing home data, etc. to ascertain diagnosis avoids missing large numbers of cases, possibly not missing at random, which likely occurs in studies using diagnoses based on hospital admission records.

Some of our models showed a protective effect of $\mathrm{O}_{3}$. However, when we compare results in Fig. 2, we see that in single-pollutant models the effect estimate for $\mathrm{O}_{3}$ was null, whereas in bi-pollutant models with either $\mathrm{PM}_{2.5}$ or $\mathrm{NO}_{2}$, the effect size for $\mathrm{O}_{3}$ was pushed below the null (albeit not significantly) and only in the tri-pollutant model was it protective at the conventional 0.05 level. Moreover, in the bi-pollutant models with $\mathrm{O}_{3}$, the effect sizes for $\mathrm{PM}_{2.5}$ and $\mathrm{NO}_{2}$ increased from their level in the single-pollutant models. We interpret this as evidence that there is no effect of $\mathrm{O}_{3}$, and the protective effect seen in the tri-pollutant model may be due to collinearity, even though the correlations between pollutants are moderate. The Pearson correlation between 5-year moving average $\mathrm{NO}_{2}$ and warm-season $\mathrm{O}_{3}$ is positive, which is inconsistent with previous studies within cities that show a negative correlation ${ }^{24}$. One possibility is that our study included exposure estimates with full coverage including both urban and rural areas, unlike previous studies that only focused on cities. The chemical regime of $\mathrm{O}_{3}$ formation in rural areas is typically $\mathrm{NO}_{\mathrm{x}}$-limited, and a positive correlation between $\mathrm{O}_{3}$ and $\mathrm{NO}_{2}$ is expected.

Higher effect estimates were observed for more populated areas (Fig. 4). $\mathrm{PM}_{2.5}$ in urban areas consists of more ultrafine particles, such as soot and metals, mainly emitted from traffic sources. Numerous studies have shown that traffic-related air pollution can be a risk factor for dementia and $\mathrm{AD}^{25-27}$. Ultrafine particles can move up the olfactory nerve into the brain directly and can penetrate into the blood and reach the blood-brain barrier ${ }^{28}$. Although $\mathrm{NO}_{2}$ is a single species and the composition is uniform everywhere, its spatial pattern shows a high correlation with major highways and cities, and the $\mathrm{NO}_{2}$ concentration may serve as a proxy for other traffic/urban air pollutants, which might have high neurotoxicity.

Our results are broadly consistent with developing literature, which shows relatively consistent effects for $\mathrm{PM}_{2.5}$ and $\mathrm{NO}_{2}$, but less consistent for $\mathrm{O}_{3}$. We observed an $\mathrm{HR}$ of 1.06 for dementia and an $\mathrm{HR}$ of 1.08 for $\mathrm{AD}$ per $3.2 \mu \mathrm{g} / \mathrm{m}^{3}$ increase in annual $\mathrm{PM}_{2.5}$ in singlepollutant models, i.e., equivalent to an $\mathrm{HR}$ of 1.10 and an $\mathrm{HR}$ of 1.13 per $5 \mu \mathrm{g} / \mathrm{m}^{3}$ increase in $\mathrm{PM}_{2.5}$. These values can be compared with our previous Medicare cohort study using hospitalizations ${ }^{7}$, reporting an $\mathrm{HR}$ of 1.06 for dementia and an $\mathrm{HR}$ of 1.17 for $\mathrm{AD}$ per $5 \mu \mathrm{g} / \mathrm{m}^{3}$ increase in annual $\mathrm{PM}_{2.5}$. A cohort study conducted in Ontario, Canada by Chen et al. ${ }^{29}$ simultaneously accessed the effects of $\mathrm{PM}_{2.5}$, $\mathrm{NO}_{2}$, and $\mathrm{O}_{3}$ on dementia risks, and they also found significant associations with $\mathrm{PM}_{2.5}$ and $\mathrm{NO}_{2}$, but not $\mathrm{O}_{3}$. Recent 2018 and 2020 Lancet Commission overviews of modifiable environmental agents 
associated with disease noted a possible association between air pollutants and dementia, but noted the evidence still preliminary ${ }^{1,30}$.

The epidemiologic findings are supported by brain imaging and toxicologic studies. Regarding brain imaging, Shaffer et al. ${ }^{31}$ have found associations between $\mathrm{PM}_{2.5}$ and $\mathrm{AD}$ neuropathology upon autopsy, while Laccarino et al. ${ }^{32}$ found an association between $\mathrm{PM}_{2.5}$ and positive positron emission tomography (PET) scans for amyloid. Younan et al. ${ }^{33}$ followed 1000 women and found increased cognitive decline on immediate memory/new learning, and increased MRIdetermined risk for future $\mathrm{AD}$ using a neuroanatomical risk score. These recent findings support earlier neuroanatomical associations found by others ${ }^{34,35}$. Toxicological studies support several plausible biological mechanisms. $\mathrm{PM}_{2.5}$ has been consistently linked to oxidative stress, neuroinflammation, systemic inflammation, and all of which, in turn, have been reported as key pathways to $\mathrm{AD}$ pathogenesis $26,34,36$. Magnetite nanoparticles from combustion processes have been discovered in the human brain, indicating that particles from urban air pollution can reach the blood-brain barrier (e.g., by interacting with dysfunctional cells) ${ }^{37}$.

The strongest relationship we found with both endpoints was for $\mathrm{PM}_{2.5}$ among the three pollutants. If the US $\mathrm{PM}_{2.5}$ levels could be lowered by $3.2 \mu \mathrm{g} / \mathrm{m}^{3}$, which is the IQR, then the attributable fraction $(\mathrm{AF})$ of dementia and $\mathrm{AD}$ due to current exposure levels, based on our main results from tri-pollutant models assuming a linear relationship, would be $6 \%$ and $7 \%$, respectively. Namely, if there is a causal relationship (although we acknowledge this is not possible in the present associative study), this would suggest an estimated $6 \%$ of dementia cases and $7 \%$ of $\mathrm{AD}$ cases would potentially be avoided if $\mathrm{PM}_{2.5}$ levels decreased by $3.2 \mu \mathrm{g} / \mathrm{m}^{3}$, which is approximately the difference between our large cities like New York and Chicago and smaller cities like Portland, Buffalo, or Baltimore ${ }^{38}$. Likewise, if the US $\mathrm{NO}_{2}$ levels could be reduced by $11.6 \mathrm{ppb}$ (IQR), an estimated $2 \%$ of dementia cases and $3 \%$ of $\mathrm{AD}$ cases would be avoided assuming a causal relationship.

Our study has several strengths. To our knowledge, this is the first nationwide, population-based cohort study that focuses on the simultaneous health effects of $\mathrm{PM}_{2.5}, \mathrm{NO}_{2}$, and $\mathrm{O}_{3}$ on dementia and $\mathrm{AD}$. The large sample size gives us ample power to detect effects even though they are small, which is often the case in environmental studies. Second, the use of Medicare claims data that include doctor's visits rather than restricting the data to hospitalizations is likely to include many more cases, given that many cases are never hospitalized, and also cases which are diagnosed earlier and hence better reflect incidence. Evidence can be found by comparing recent data in another paper about dementia and $\mathrm{AD}$ hospitalization in Medicare data ${ }^{7}$, to the data in the current paper. To allow for a fair comparison, we used the same inclusion/exclusion criteria and restricted to the same time period (2000-2016) and geographic region (i.e., the lower 48 states), and we found that using just hospitalization missed nearly $90 \%$ of dementia cases and $60 \%$ of AD cases, compared to using our current data including doctor's visits (Supplementary Table 9). Third, we used a conservative method by requiring a 5 -year "clean" period and restricting the analysis to subjects with continuous enrollment in Medicare Fee-for-Service (FFS), and Part A (hospital insurance) and Part B (medical insurance) programs throughout the study period, which can ensure that cases were newly diagnosed and thus better approximate incidence. At last, we were able to control for a large number of individual- and neighborhood-level covariates. The inclusion of comorbidities had a negligible effect on our results, suggesting that they are unlikely mediators in our studied associations. However, a formal mediation analysis would be important to confirm these findings.

Despite these advantages, some key limitations should be noted. One limitation, typical of using administrative records to identify disease, is potential misclassification of the outcome. AD cases in our database represented only $\sim 40 \%$ of the dementia cases, suggesting important under-ascertainment of $\mathrm{AD}$, given that $\mathrm{AD}$ represents $\sim 60-80 \%$ of dementia cases ${ }^{2}$. This percentage is quite similar to the findings of Goodman et al. ${ }^{39}$, who found that $\mathrm{AD}$ represented $44 \%$ of all dementia diagnoses in Medicare data in 2013, including both hospitalizations and doctor visits. It is likely that a large number of our dementia cases, who show no $\mathrm{AD}$ diagnosis in Medicare, actually had $\mathrm{AD}$, but physicians did not feel confident to make the more specific diagnosis. This is supported by the findings of Taylor et al. (2009), who compared Medicare data to clinical diagnoses considered as the gold standard, and found that the sensitivity of dementia was 0.85 but was considerably lower, 0.65 , for AD. At last, given the long insidious onset of dementia and lack of information on the stage of dementia at diagnosis, our study may mismeasure the year of onset of dementia, though we applied the 5-year "clean" period as inclusion criteria to reduce the bias.

We have assumed that outcome misclassification is nondifferential (conditionally independent of exposure to air pollutants, conditional on confounders); there are no data indicating otherwise. We have conducted two types of sensitivity analyses to adjust for such misclassification of classifying dementia or $\mathrm{AD}$ cases as without dementia or $\mathrm{AD}$ (false negative, or 1-sensitivity), and the misclassification of non-dementia, non-AD subjects to one of the diseases (false positive, or 1-specificity). Both these methods of adjustment for false negatives and false positives were in agreement that our results were likely to under-estimate the true HRs for $\mathrm{PM}_{2.5}$ and $\mathrm{NO}_{2}$ for both dementia and $\mathrm{AD}$.

Another limitation of our study is the potential exposure error and its spatial pattern, although the exposure prediction model we used has excellent predictive accuracy ${ }^{16-18}$. Using larger scale ambient air pollution assigned to individuals has been shown to have a net bias towards the null, consistent with non-differential measurement error, which reflects some degree classical type of error $^{40-42}$. In addition, our study is subject to unmeasured and residual confounding. While we were able to control for a number of potential confounders at the neighborhood level, we had no individual-level data on SES and education, a limitation implying some mismeasurement of confounders, which may have biased our results (moderately, given that these unmeasured confounders are not likely to act as very strong risk factors for dementia), in an unknown direction. Furthermore, we only studied the Medicare FFS population who enrolled in both Part A and Part B programs, precluding generalizability to the entire US elderly population. Further work is also needed to determine if the association is generalizable in other countries.

Our study provides evidence that long-term exposure to $\mathrm{PM}_{2.5}$ mass is associated with increased ADRD incidence. Future studies of air pollution and dementia in other countries, including lowto-middle-income countries on which there are currently few studies, will be important. Understanding the potential bias and unmeasured confounding, given the limitations of observational studies, is encouraged. Examining the role of specific pollutant components in ADRD may also be important because different components of $\mathrm{PM}_{2.5}$ (e.g., metals, elemental carbon, organic carbon, sulfate, and nitrate) and different sources of $\mathrm{PM}_{2.5}$ (e.g., traffic, industrial, cooking, and biomass burning) may have different neurotoxicities. A better understanding of componentspecific and source-specific effects of $\mathrm{PM}_{2.5}$ on ADRD could potentially inform pollution control policies on specific sources.

\section{Methods}

Study population. Data were drawn from the Medicare denominator file and the Medicare Chronic Conditions Warehouse (CCW), both from the Centers for Medicare and Medicaid Services (CMS). In the U.S., people are eligible to enter the 
Medicare program after they turn 65 years of age. The denominator file (i.e., the enrollment file) contains enrollment records for all Medicare beneficiaries, including age, sex, race, Medicaid eligibility (a proxy for SES), the date of death (if any), and ZIP code of residence. Age, Medicaid eligibility, and ZIP code of residence are updated annually. CCW provides the date of the first occurrence with dementia or $\mathrm{AD}$ diagnosis code across the available Medicare claims. Based on these two Medicare databases, we constructed an open cohort including all Medicare beneficiaries aged 65 and over who were always enrolled (1) in Medicare Feefor-Service program; and (2) in both Medicare Part A (hospital insurance) and Part B (medical insurance) in the contiguous United States between 2000 and 2018. These criteria excluded those with any time in Medicare Advantage (HMO) over the study period since claim records are not available for these beneficiaries and excluded those only enrolled in Medicare Part A or Part B. If we relaxed these restrictions to broaden the cohort, the chance of missing dementia or $\mathrm{AD}$ cases among those additional people brought into the analysis would be high.

We created separate data sets for dementia and $\mathrm{AD}$. For each cohort, we further required a "clean" period of 5 years after enrollment, during which there were no diagnosis codes for dementia or AD. For example, a participant entering Medicare in 2005 would be required to be dementia-free until 2010; follow-up for disease incidence began only then. By removing potentially prevalent cases in their first five years of follow-up, a diagnosis after that "clean" period more likely approximates disease "incidence". We considered that 5 years was a reasonable period to ensure that a person truly was not demented prior to the Medicare diagnosis; however, we also explored a 10 -year clean period in sensitivity analyses. Therefore, study subjects entered the cohort on January 1st of the year following the "clean" period and were followed until the first diagnosis of the outcome of interest across all Medicare claims, death, or end of followup. We excluded this 5-year clean period from follow-up time to avoid immortal time bias. A schematic diagram of the cohort selection criteria is illustrated in Supplementary Fig. 1. Our research is approved by Emory's IRB (\#STUDY00000316) and the Centers for Medicare \& Medicaid Services (CMS) under the data use agreement (\#RSCH-202055733). The Medicare dataset was stored and analyzed in Emory Rollins School secure cluster environment (HPC), with Health Insurance Portability and Accountability Act (HIPAA) compliance.

Data management and maintenance of confidentiality. Emory provides and operates the secure cluster environment HPC certified for use with confidential health records data (e.g., Medicare) storage and analysis and safeguard the data in compliance with the HIPAA security rule. The CMS data files are stored on the secure servers at Emory Rollins School of Public Health. All the participating research members are granted Emory-affiliated user accounts with which to access processed data for the duration of the project. No transfer of CMS data from Emory systems is allowed for any user. In addition, a technical data scientist at Emory is monitoring the activities of the user accounts and manually inspecting any derived data and output from analyses. When an analysis is complete, data access will be removed for that investigator.

Outcome classification. The primary outcomes of interest for this study were time to (1) all-cause dementia and (2) AD subtype. CCW includes pre-defined indicators for dementia and $\mathrm{AD}$, which are identified using an algorithm ${ }^{43}$ that incorporates information from all available Medicare claims (such as inpatient and outpatient claims, Carrier file, skilled nursing facility, and home health-care claims) indicating that an individual was diagnosed with dementia or AD (ICD-codes provided in Supplemental Material ${ }^{43}$ ). This algorithm applied by Medicare to define dementia and $\mathrm{AD}$ is primarily based on Taylor et al. ${ }^{44}$ and Taylor et al. ${ }^{45}$. CCW provides the date of the first occurrence with the dementia or $\mathrm{AD}$ diagnosis code. In the dementia cohort, the outcome dementia was defined as the first occurrence of a diagnosis code of dementia, while for the $\mathrm{AD}$ cohort, $\mathrm{AD}$ was defined as either (1) the first occurrence of a diagnosis code of $\mathrm{AD}$ with no prior diagnosis of dementia, or (2) the first occurrence of a diagnosis code of dementia when there was a subsequent diagnosis code of $\mathrm{AD}$ (under the supposition that the original dementia diagnosis was probably $\mathrm{AD}$, given the subsequent $\mathrm{AD}$ diagnosis).

Exposure assessment. High-resolution daily ambient $\mathrm{PM}_{2.5}$ (24-hour average), $\mathrm{NO}_{2}$ (1-hour maximum), and $\mathrm{O}_{3}$ (8-hour maximum) concentrations at 1-km spatial resolution for the entire United States were derived using spatiotemporal ensemble models that integrated three different machine learning algorithms, including neural networks, random forest, and gradient boosting. The ensemblebased model was calibrated using hundreds of predictors, including satellite measurements, chemical transport model simulations, land-use terms, meteorological variables, and monitoring measurements from the Environmental Protection Agency (EPA) Air Quality Systems (AQS). This ensemble learning approach yielded strong prediction model performance for each pollutant, with an average cross-validated coefficient of determination $\left(R^{2}\right)$ of $0.89,0.84$, and 0.86 for annual mean $\mathrm{PM}_{2.5}$, annual mean maximum 1-hour $\mathrm{NO}_{2}$, and warm-season (May to October) mean maximum 8-hour $\mathrm{O}_{3}$, respectively ${ }^{16-18}$. We averaged these $1-\mathrm{km}$ resolution predictions for each pollutant at the ZIP code scale across each year ${ }^{46}$, because ZIP Code is the smallest level of geography in the Medicare data. We used the annual averages in each ZIP code, for each calendar year, as the exposure estimates for each Medicare beneficiary according to the ZIP code of residence. We calculated time-varying 5-year moving averages of exposure for each follow-up year for each subject. For example, air pollution estimates for someone entering Medicare in 2005 and being diagnosed with dementia in 2013 would include the average air pollution over the period from 2008-2013 and linked to the last followup year (i.e., 2013). All dementia and $\mathrm{AD}$ events were linked to exposures averaged over 5 years prior to diagnosis, and any annual residential mobility changes by ZIP code were taken into account, based on their yearly residence in the Medicare database. All high-resolution $\mathrm{PM}_{2.5}, \mathrm{NO}_{2}$, and $\mathrm{O}_{3}$ data used in this study were available from 2000 to 2016 . For the follow-up year of 2017, we assigned the multiple-year average exposure during 2012-2016 to each subject (since 2017 exposure is not available); for the follow-up year of 2018, we assigned the multipleyear average exposure during 2013-2016 to each subject.

Covariates. Individual-level age at entry, sex, race, and Medicaid eligibility were obtained from the Medicare denominator file. We also obtained neighborhoodlevel covariates in our study. These included ZIP code-level SES variables (population density, \% Black population, education, median household income, \% owner-occupied housing units, and \% population above 65 years of age living below the poverty line), county-level behavioral risk factors (smoking rate and body mass index) and health-care capacity variables (number of hospitals and active medical doctors), as well as a geographical region. Specifically, SES variables were obtained from the 2000 U.S. Census ${ }^{47}, 2010$ U.S. Census ${ }^{48}$, and the American Community Survey for $2005-2012 ; 49$ behavioral risk factors were obtained from the Behavioral Risk Factor Surveillance System (BRFSS) between 2000 and 2016; ${ }^{50}$ and healthcare capacity data were obtained from 2010, 2015, and 2018 American Hospital Association Annual Survey Database ${ }^{51}$. We linearly interpolated or extrapolated any missing data based on the data available ${ }^{52}$. Data were also available for comorbidities (diabetes, heart failure, stroke, hypertension) in CCW. These covariates have been associated previously with ADRD and may be associated with air pollution, and hence were candidate confounders to be included in models 53,54 .

Statistical analysis. We fit a series of stratified Cox proportional hazards models with a generalized estimating equation (GEE) ${ }^{55}$ to estimate the associations between long-term exposure to $\mathrm{PM}_{2.5}, \mathrm{NO}_{2}$, and $\mathrm{O}_{3}$ on dementia or $\mathrm{AD}$ among the elderly, where the coefficient for the exposure variable was the parameter of interest, and years of follow-up was the time scale. Specifically, we fit singlepollutant, bi-pollutant, and tri-pollutant models and estimated HRs per interquartile-range (IQR) increase in the 5-year average of the annual $\mathrm{PM}_{2.5}, \mathrm{NO}_{2}$, and warm-season $\mathrm{O}_{3}$ concentrations in the 5 years prior to diagnosis. All three pollutants are of interest because some prior literature has shown associations between each of them and dementia $7,25,29,56$. GEE was used to adjust for residual autocorrelation within ZIP code with the use of robust standard errors (and 95\% CIs). To allow for flexible strata-specific baseline hazard functions, we stratified all models on four individual characteristics, including sex, race (white, Black, other), Medicaid eligibility, and 1-year categories of age at study entry. To adjust for potential confounding, we included neighborhood-level SES, behavioral risk factors, and health-care capacity variables in our analyses. Potential residual temporal and spatial trends were controlled by respectively including a linear term for calendar years and indicator variables for the geographical region ${ }^{7}$.

To assess the shape of the C-R relationship between each air pollutant and dementia or $\mathrm{AD}$, we respectively fit penalized splines ${ }^{7}$ for $\mathrm{PM}_{2.5}, \mathrm{NO}_{2}$, and $\mathrm{O}_{3}$, adjusting for all covariates included in the tri-pollutant models. To identify subpopulations who might be more vulnerable than others, we assessed potential effect modification by sex, race, Medicaid eligibility, age groups (aged 75+ vs. below 75), and urbanicity (quartiles of population density) on the multiplicative scale by including interaction terms between these potential modifiers and pollutants.

In addition, we estimated the attributable fraction $(\mathrm{AF})$ of dementia and $\mathrm{AD}$ cases due to $\mathrm{PM}_{2.5}$ and $\mathrm{NO}_{2}$ air pollution, for those in the US exposed to an additional IQR of $\mathrm{PM}_{2.5}$ (a difference of $3.2 \mu \mathrm{g} / \mathrm{m}^{3}$ ) and $\mathrm{NO}_{2}$ (a difference of $11.6 \mathrm{ppb}$ ), beyond current levels in US cities with relatively low exposure (i.e., $7 \mu \mathrm{g} / \mathrm{m}^{3}$ for $\mathrm{PM}_{2.5}$ and $4 \mathrm{ppb}$ for $\mathrm{NO}_{2}$, the counterfactual ${ }^{38}$, using results from the multi-pollutant model, and using standard AF calculations when the entire population is exposed (RR-1)/RR (see Steenland and Armstrong ${ }^{57}$ ).

We conducted a series of sensitivity analyses to test the robustness of our main findings. First, we repeated the analyses using a "clean" period of 10 years, i.e., thinking that excluding cases with a diagnosis during their first 10 years of enrollment would increase the probability that we are capturing the first diagnosis and thus more closely estimating disease incidence, albeit at the cost of a smaller number of years of follow-up and cases. Second, using this new subcohort, we assessed alternative exposure time windows by comparing the results using different lags (0-, 1-, 5-, and 10-year lags), in which exposure was assigned either as the annual exposure at 10 years prior to case (or the risk set for given cases), or 5 years prior, or 1 or 0 years prior. We posit that if a shorter lag between exposure and disease fits the data best, assuming the association is causal, would imply an acceleration of an existing process (e.g., dementia progression) by air pollution, whereas a longer lag might indicate the air pollution has an effect in more initial stages of neurodegeneration (e.g., involved in the onset of dementia). In addition, to evaluate whether the associations we observe can be attributed to comorbidities 
also linked to air pollution, we additionally adjusted for the comorbidities (including diabetes, hypertension, stroke, and heart failure), and also restricted analyses to subjects without the comorbidities. Finally, we conducted analyses to estimate the effect of possible outcome misclassification in two ways. First, we fit linear regression models for the rate of dementia or $\mathrm{AD}$ (events/person-time) with a GEE, which in theory should target an approximately unbiased estimate of the additive effect ${ }^{58}$. Second, we considered the possible effect of outcome misclassification following methods similar to those described by Fox et al. ${ }^{59}$. We obtained estimates of misclassification parameters from Taylor et al. ${ }^{45}$ and adjusted the observed outcomes for each stratum to match up with the expected true values given pre-specified values for sensitivity and specificity for the outcome classification (details provided in Supplemental Material).

All computational analyses ${ }^{60}$ were run on the Rollins HPC Cluster at Emory University. R software, version 4.0.2, was used for all analyses. A two-sided $P<0.05$ was considered statistically significant.

Reporting summary. Further information on research design is available in the Nature Research Reporting Summary linked to this article.

\section{Data availability}

Ensemble-based $\mathrm{PM}_{2.5}$ data that support the findings of this study are available from https://doi.org/10.7927/0rvr-4538, $\mathrm{NO}_{2}$ and $\mathrm{O}_{3}$ data are available from https://doi.org/ 10.6084/m9.figshare.16834390. Behavioral risk factors are available from https:// www.cdc.gov/brfss/annual_data/annual_data.html; SES data are available from https:// www.census.gov/data/datasets/2000/dec/summary-file-3.html, https://www.census.gov/ data/datasets/2010/dec/summary-file-1.html, and https://www.census.gov/data/ developers/data-sets/acs-1year.html; health-care capacity data are available from https:// data.hrsa.gov/topics/health-workforce/ahrf. The rules governing the Medicare dataset prohibit any sharing of the health datasets being used for our epidemiologic research Restricted by our Data Use Agreement with the US Centers for Medicare \& Medicaid Services, the Medicare data that support the findings of this study are neither sharable nor publicly available. Academic and non-profit researchers who are interested in using Medicare data should contact the US Centers for Medicare \& Medicaid Services directly to obtain their own datasets upon completion of a Data Use Agreement. Source data are provided with this paper.

\section{Code availability}

The $\mathrm{R}$ codes for the epidemiological analyses are publicly available from https://doi.org/ $10.6084 / \mathrm{m} 9$.figshare.16843528.

Received: 25 May 2021; Accepted: 1 November 2021;

Published online: 19 November 2021

\section{References}

1. Livingston, G. et al. Dementia prevention, intervention, and care. Lancet 390, 2673-2734 (2017).

2. Heron, M. P. Deaths: leading causes for 2017. Natl. Vital. Stat. Rep. 68, 1-77 (2019).

3. Khachaturian, Z. S., Khachaturian, A. S. \& Thies, W. The draft "National Plan" to address Alzheimer's disease-National Alzheimer's Project Act (NAPA). Alzheimer's Dement. 8, 234-236 (2012).

4. Peters, R. et al. Air pollution and dementia: a systematic review. J. Alzheimer's Dis. 70, S145-S163 (2019).

5. Fu, P. \& Yung, K. K. L. Air pollution and Alzheimer's disease: a systematic review and meta-analysis. J. Alzheimer's Dis. 77, 701-714 (2020).

6. van Wijngaarden, E. et al. Neurodegenerative hospital admissions and long term exposure to ambient fine particle air pollution. Ann. Epidemiol. 54, 79-86. e74 (2021).

7. Shi, L. et al. Long-term effects of PM2. 5 on neurological disorders in the American Medicare population: a longitudinal cohort study. Lancet Planet. Health 4, e557-e565 (2020).

8. Smargiassi, A. et al. Exposure to ambient air pollutants and the onset of dementia in Québec, Canada. Environ. Res. 190, 109870 (2020).

9. Grande, G., Ljungman, P. L., Eneroth, K., Bellander, T. \& Rizzuto, D. Association between cardiovascular disease and long-term exposure to air pollution with the risk of dementia. JAMA Neurol. 77, 801-809 (2020).

10. Ilango, S. D. et al. The role of cardiovascular disease in the relationship between air pollution and incident dementia: a population-based cohort study. Int. J. Epidemiol. 49, 36-44 (2020).

11. Lee, M., Schwartz, J., Wang, Y., Dominici, F. \& Zanobetti, A. Long-term effect of fine particulate matter on hospitalization with dementia. Environ. Pollut. 254, 112926 (2019).
12. Mortamais, M. et al. Long-term exposure to ambient air pollution and risk of dementia: results of the prospective three-city study. Environ. Int. 148, 106376 (2021).

13. Nunez, Y. et al. Fine particle exposure and clinical aggravation in neurodegenerative diseases in New York state. Environ. Health Perspect. 129, 027003 (2021).

14. Sullivan, K. J. et al. Ambient fine particulate matter exposure and incident mild cognitive impairment and dementia. J. Am. Geriatr. Soc. 69, 2185-2194 (2021).

15. Jack, C. R. Jr et al. Hypothetical model of dynamic biomarkers of the Alzheimer's pathological cascade. Lancet Neurol. 9, 119-128 (2010).

16. Di, Q. et al. An ensemble-based model of PM2. 5 concentration across the contiguous United States with high spatiotemporal resolution. Environ. Int. 130, 104909 (2019).

17. Di, Q. et al. Assessing $\mathrm{NO}_{2}$ concentration and model uncertainty with high spatiotemporal resolution across the contiguous united states using ensemble model averaging. Environ. Sci. Technol. 54, 1372-1384 (2019).

18. Requia, W. J. et al. An ensemble learning approach for estimating high spatiotemporal resolution of ground-level ozone in the contiguous United States. Environ. Sci. Technol. 54, 11037-11047 (2020).

19. Semega, J. L., Fontenot, K. R. \& Kollar, M. A. Income and poverty in the United States: 2016. Current Population Reports (2017).

20. Jacob, D. J. Introduction to atmospheric chemistry. (Princeton University Press, 1999).

21. Jerrett, M. et al. Long-term ozone exposure and mortality. N. Engl. J. Med 360, 1085-1095 (2009).

22. Heusinkveld, H. J. et al. Neurodegenerative and neurological disorders by small inhaled particles. Neurotoxicology 56, 94-106 (2016).

23. Peters, R., Mudway, I., Booth, A., Peters, J. \& Anstey, K. J. Putting fine particulate matter and dementia in the wider context of noncommunicable disease: where are we now and what should we do next: a systematic review. Neuroepidemiology 55, 253-265 (2021).

24. Williams, M., Atkinson, R., Anderson, H. \& Kelly, F. Associations between daily mortality in London and combined oxidant capacity, ozone and nitrogen dioxide. Air Qual. Atmos. Health 7, 407-414 (2014).

25. Chen, $H$. et al. Living near major roads and the incidence of dementia, Parkinson's disease, and multiple sclerosis: a population-based cohort study. Lancet 389, 718-726 (2017).

26. Ranft, U., Schikowski, T., Sugiri, D., Krutmann, J. \& Krämer, U. Long-term exposure to traffic-related particulate matter impairs cognitive function in the elderly. Environ. Res. 109, 1004-1011 (2009).

27. Tham, R. \& Schikowski, T. The role of traffic-related air pollution on neurodegenerative diseases in older people: an epidemiological perspective. $J$. Alzheimer's Dis. 79, 949-959 (2020).

28. Oberdörster, G. et al. Translocation of inhaled ultrafine particles to the brain. Inhal. Toxicol. 16, 437-445 (2004).

29. Chen, H. et al. Exposure to ambient air pollution and the incidence of dementia: a population-based cohort study. Environ. Int. 108, 271-277 (2017).

30. Landrigan, P. J. et al. The Lancet Commission on pollution and health. Lancet 391, 462-512 (2018).

31. Shaffer, R. M. et al. Fine particulate matter and markers of Alzheimer's disease neuropathology at autopsy in a community-based cohort. J. Alzheimer's Dis. 79, 1761-1773 (2021)

32. Iaccarino, L. et al. Association between ambient air pollution and amyloid positron emission tomography positivity in older adults with cognitive impairment. JAMA Neurol. 78, 197-207 (2021).

33. Younan, D. et al. Particulate matter and episodic memory decline mediated by early neuroanatomic biomarkers of Alzheimer's disease. Brain 143, 289-302 (2020).

34. Calderón-Garcidueñas, L. et al. Long-term air pollution exposure is associated with neuroinflammation, an altered innate immune response, disruption of the blood-brain barrier, ultrafine particulate deposition, and accumulation of amyloid $\beta-42$ and $\alpha$-synuclein in children and young adults. Toxicol. Pathol. 36, 289-310 (2008).

35. Cacciottolo, M. et al. Particulate air pollutants, APOE alleles and their contributions to cognitive impairment in older women and to amyloidogenesis in experimental models. Transl. Psychiatry 7, e1022-e1022 (2017).

36. Levesque, S., Surace, M. J., McDonald, J. \& Block, M. L. Air pollution \& the brain subchronic diesel exhaust exposure causes neuroinflammation and elevates early markers of neurodegenerative disease. J. Neuroinflamm. 8, 1-10 (2011).

37. Maher, B. A. et al. Magnetite pollution nanoparticles in the human brain. Proc. Natl. Acad. Sci. 113, 10797-10801 (2016).

38. USEPA. Air Quality - Cities and Counties, https://www.epa.gov/air-trends/airquality-cities-and-counties (2020).

39. Goodman, R. A. et al. Prevalence of dementia subtypes in United States Medicare fee-for-service beneficiaries, 2011-2013. Alzheimer's Dement. 13, 28-37 (2017).

40. Kioumourtzoglou, M.-A. et al. Exposure measurement error in PM 2.5 health effects studies: a pooled analysis of eight personal exposure validation studies. Environ. Health 13, 2 (2014). 
41. $\mathrm{Wu}, \mathrm{X}$. et al. Causal inference in the context of an error prone exposure: air pollution and mortality. Ann. Appl. Stat. 13, 520-547 (2019).

42. Zeger, S. L. et al. Exposure measurement error in time-series studies of air pollution: concepts and consequences. Environ. Health Perspect. 108, 419-426 (2000).

43. Chronic Conditions Data Warehouse. Condition Categories, https:// www2.ccwdata.org/web/guest/condition-categories (2021).

44. Taylor, D. H. Jr, Fillenbaum, G. G. \& Ezell, M. E. The accuracy of medicare claims data in identifying Alzheimer's disease. J. Clin. Epidemiol. 55, 929-937 (2002).

45. Taylor, D. H. Jr, Østbye, T., Langa, K. M., Weir, D. \& Plassman, B. L. The accuracy of Medicare claims as an epidemiological tool: the case of dementia revisited. J. Alzheimer's Dis. 17, 807-815 (2009).

46. Shi, L. Yearly data for NO2 and warm season ozone in the US, https://doi.org/ 10.6084/m9.figshare.16834390 (2021).

47. Census, U. Summary File 3 Dataset, https://www.census.gov/data/datasets/ 2000/dec/summary-file-3.html (2002).

48. Census, U. Summary File 1 Dataset, https:/www.census.gov/data/datasets/ 2010/dec/summary-file-1.html (2011)

49. Census, U. American Community Survey 1-Year Data (2005-2019), https:// www.census.gov/data/developers/data-sets/acs-1year.html (2020).

50. CDC, U. Behavioral Risk Factor Surveillance System https://www.cdc.gov/ brfss/annual_data/annual_data.htm (2020).

51. Area Health Resources Files, https://data.hrsa.gov/topics/health-workforce/ ahrf (2019)

52. Junninen, H., Niska, H., Tuppurainen, K., Ruuskanen, J. \& Kolehmainen, M. Methods for imputation of missing values in air quality data sets. Atmos. Environ. 38, 2895-2907 (2004).

53. Hersi, M. et al. Risk factors associated with the onset and progression of Alzheimer's disease: a systematic review of the evidence. Neurotoxicology 61, 143-187 (2017).

54. Anstey, K. J., Ee, N., Eramudugolla, R., Jagger, C. \& Peters, R. A systematic review of meta-analyses that evaluate risk factors for dementia to evaluate the quantity, quality, and global representativeness of evidence. J. Alzheimer's Dis. 70, S165-S186 (2019).

55. Zhang, X. Generalized estimating equations for clustered survival data. Retrospective Theses and Dissertations, 3063. https://lib.dr.iastate.edu/rtd/3063 (2006).

56. Cerza, F. et al. Long-term exposure to air pollution and hospitalization for dementia in the Rome longitudinal study. Environ. Health 18, 72 (2019).

57. Steenland, K. \& Armstrong, B. An overview of methods for calculating the burden of disease due to specific risk factors. Epidemiology 17, 512-519 (2006).

58. Hutcheon, J. A., Chiolero, A. \& Hanley, J. A. Random measurement error and regression dilution bias. BMJ 340, c2289 (2010).

59. Fox, M. P., Lash, T. L. \& Greenland, S. A method to automate probabilistic sensitivity analyses of misclassified binary variables. Int. J. Epidemiol. 34, 1370-1376 (2005).

60. Shi, L. $R$ code for epidemiological analyses for ADRD, https://doi.org/10.6084/ m9.figshare.16843528 (2021).

\section{Acknowledgements}

This study was supported by the National Institute on Aging (NIA/NIH R01 AG074357, L.S.), the HERCULES Center P30 ES019776 (L.S.), and the Goizueta Alzheimer's Disease Research Center (ADRC) of Emory University (P50 AG025688, L.S.). The views expressed in this article are those of the authors, and do not necessarily represent the views of the funding Agencies. The authors acknowledge Dr. Joel Schwartz's lab for providing us with access to their estimated air pollution data, and acknowledge Kimberly Lochner for fruitful discussion.

\section{Author contributions}

L.S. and K.S. designed research and directed its implementation; L.S., H.L., K.S. and R.H.L. analyzed data, L.S., P.L., Y.Z. and H.L. made the figures and tables, L.S., P.L., W.J.R. and J.S. prepared data sets; L.S., K.S., P.L., R.H.L., S.I., H.C., T.W., J.S. and R.J.W. interpreted the results. L.S. and K.S. lead the writing of the manuscript, with input from all authors.

\section{Competing interests}

The authors declare no competing interests.

\section{Additional information}

Supplementary information The online version contains supplementary material available at https://doi.org/10.1038/s41467-021-27049-2.

Correspondence and requests for materials should be addressed to Liuhua Shi.

Peer review information Nature Communications thanks Severine Sabia, Alexandra Schneider and the other, anonymous, reviewer(s) for their contribution to the peer review of this work.

Reprints and permission information is available at http://www.nature.com/reprints

Publisher's note Springer Nature remains neutral with regard to jurisdictional claims in published maps and institutional affiliations.

(c) (i) Open Access This article is licensed under a Creative Commons Attribution 4.0 International License, which permits use, sharing, adaptation, distribution and reproduction in any medium or format, as long as you give appropriate credit to the original author(s) and the source, provide a link to the Creative Commons license, and indicate if changes were made. The images or other third party material in this article are included in the article's Creative Commons license, unless indicated otherwise in a credit line to the material. If material is not included in the article's Creative Commons license and your intended use is not permitted by statutory regulation or exceeds the permitted use, you will need to obtain permission directly from the copyright holder. To view a copy of this license, visit http://creativecommons.org/ licenses/by/4.0/.

(C) The Author(s) 2021 